\title{
estimation du débit et de la nature des fuites d'un barrage en terre sur lit alluvionnaire
}

\author{
(Application au barrage de Serre-Ponçon)
}

\author{
par \\ E. Ledeuil \\ Ingénieur Arts et Métiers \\ Docteur en Mécanique des Sols
}

Exposé fait au Comité Français de Mécanique des Sols, séance du 13 novembre 1978

\section{Introduction}

L'objet de cette étude, tout en étant à caractère très général, est appuyée sur un exemple concret et vécu : le barrage de Serre-Ponçon.

La description du barrage ne sera pas reprise, seuls les traits principaux utiles pour l'exposé des faits seront cités (voir vue en plan fig. 1).

Tout barrage, et Serre-Ponçon ne fait pas exception. laisse passer de l'eau et l'Art de l'Ingénieur est de savoir par où passe cette eau et combien il en passe afin d'apprécier la valeur de l'ouvrage. Enfin par l'auscultation l'ingénieur cherchera à suivre l'évolution de ces fuites ainsi que le comportement global du barrage.

\section{Description du barrage}

2.1 La description du barrage de Serre-Ponçon a été souvent faite et la coupe type donnée en figure 2 précise les cotes principales et les numéros des piézomètres.

2.2 Le sillon profond, situé sous le barrage, rempli d'alluvions de Durance (sables et graviers) est schématisé par des coupes au droit de divers piézomètres, les sections normalement situées sous la nappe varient de $2830 \mathrm{~m}^{2}$ à $5400 \mathrm{~m}^{2}$ (voir fig. 3).

2.3 Le noyau et la coupure étanche (réalisée par injection) sont schématisés, en figure 4, avec une image des tubes d'écoulement transitant l'eau d'amont (cote 760 , à la date de l'étude) à l'aval (cote 656, soit $10 \mathrm{~m}$ sous le lit origine de la rivière, l'excavation du bassin de compensation en étant la cause).

2.4 Enfin, le rocher qui entoure le site est un calcaire marneux de bonne qualité mais parfois fracturé.

\section{Localisation des écoulements}

3.1 Sur la figure 4 l'écoulement à travers le noyau est régulier, il s'agit d'une image de synthèse issue des mesures internes et qui dépasse le "dessin d'artiste " (voir la thèse de $\mathrm{M}$. Ledeuil citée en référence bibliographique). Outre la zone de liaison entre coupure étanche et noyau, il y a en profondeur au niveau du piézomètre $n^{\circ} 8$ diverses anomalies que l'on suit et que l'on essayera d'analyser.

3.2 A l'amont la nappe est horizontale et correspond à la cote de retenue. Les piézomètres amont par l'étude de leurs retards par rapport aux variations du plan d'eau indiquent une excellente perméabilité de la recharge amont soit $10^{-3}$ à $10^{-4} \mathrm{~m} / \mathrm{s}$.

3.3 Le rocher entourant la coupure d'alluvions injectées représente sûrement le lieu privilégié des circulations d'eau amont-aval, en dépit de l'étendue de la surface du voile d'injection dans le rocher $\left(90000 \mathrm{~m}^{2}\right)$.

Les diverses mesures faites pendant la première phase de mise en eau ont montré l'intérêt de compléter le traitement d'injection du rocher et les résultats ont été sensibles. Enfin, plus tard ( 3 à 5 ans après la fin de la première mise en eau) les débits ont diminué et les diverses analyses des mesures faites laissent penser à une action de colmatage de fissures et d'étanchement superficiel par suite des dépôts de sédimentation.

3.4 A l'aval de la coupure étanche, la nappe est faiblement pentée. Cette pente sous le barrage est variable et se situe entre $1 \%$ et $2 \%$, elle est donc nettement plus faible que celle du cours de la rivière avant travaux qui était de 3 à $3,5 \%$ en moyenne.

Cette nappe aval regroupe bien entendu les divers écoulements cités ci-dessus.

Les divers piézomètres, permettent de percevoir parfois plusieurs nappes ou des écoulements, à travers le rocher ou la coupure alluviale, plus ou moins importants suivant les zones. Ils ont aussi permis l'estimation du débit global et de la nature relative des fuites. 


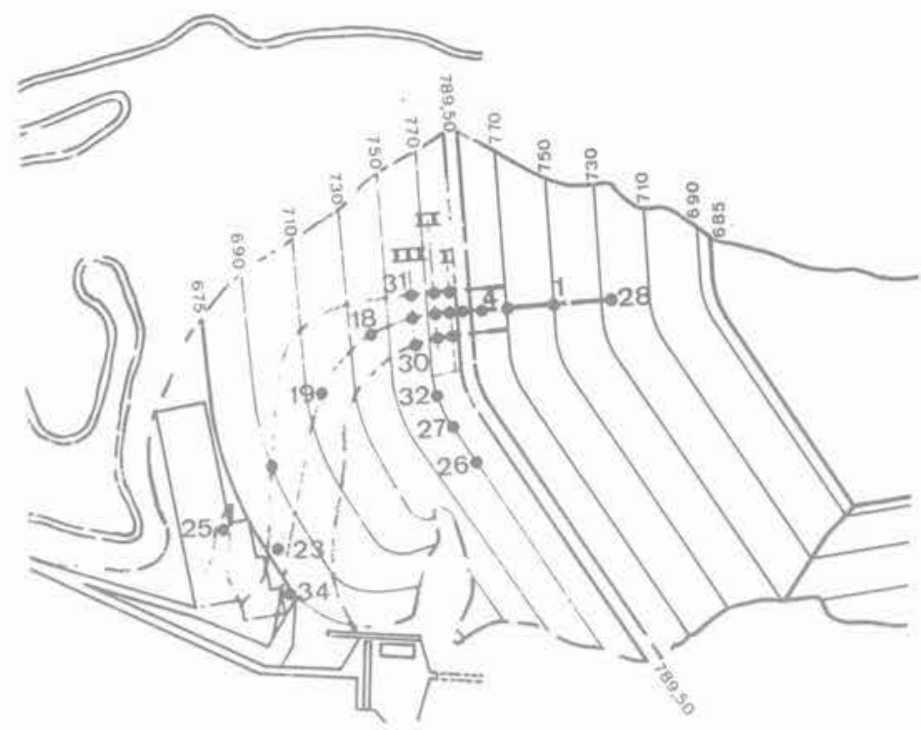

Fig. 1 Tubes piézométriques : localisation des appareils

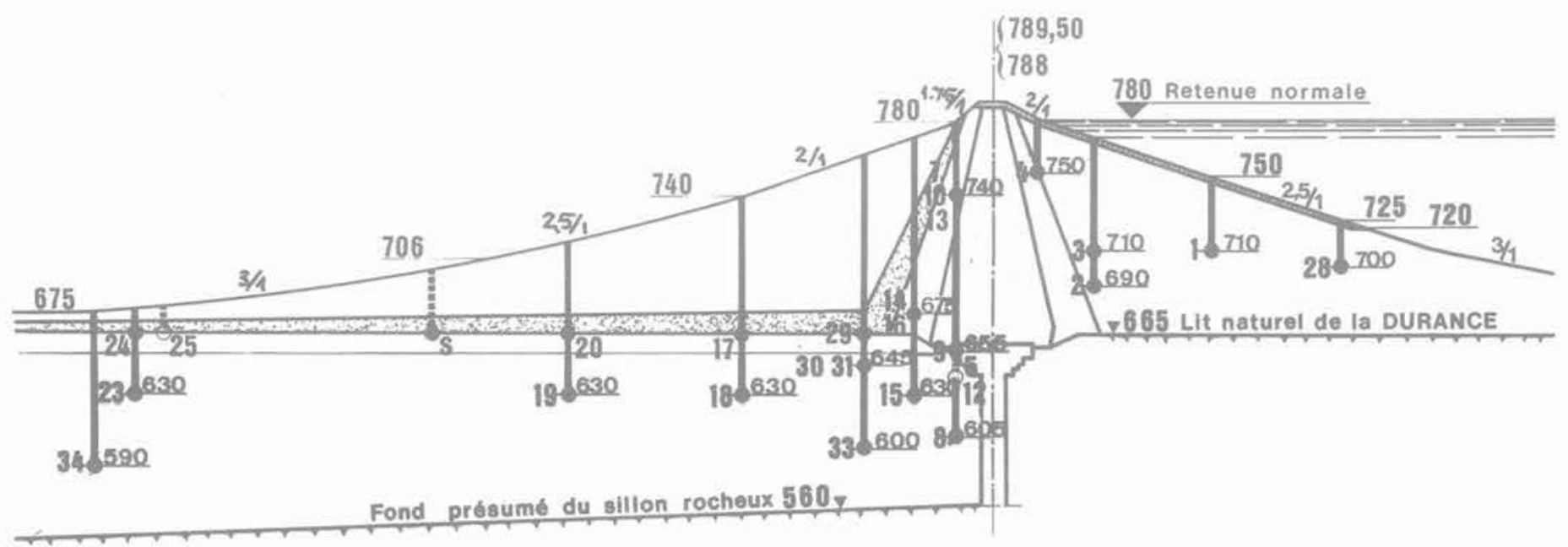

Fig. 2 Tubes piézométriques : profil dans l'axe du șillon rocheux

4 Détermination du débit et de la perméabilité du remplissage alluvial par l'étude de la variation de la nappe aval

4.1 A l'aval, la ligne de surface libre a une pente décroissante due au profil du sillon rocheux (fig. 5). Pour bien définir l'écoulement il fallait situer cette surface et définir si possible le débit qui pouvait transiter au-dessous d'elle.

On a profité d'une montée brutale de la nappe aval (provoquée par la suppression de la digue de protection de la Durance le 18 mai 1960) de l'ordre de 1,30 mètre. II ne restait plus qu'à suivre l'évolution correspondante des piézomètres aval (voir fig. 5 et 6).

4.2 Considérant les lignes piézométriques du 19 mai et du 20 mai, on remarque une élévation régulière durant ces 24 heures (les lignes sont parallèles). Le débit s'écoulant dans les fondations est lié à la pente de cette ligne piézométrique par la loi de Darcy. En effet, la pente traduit le gradient $i$ de circulation et le débit est :

$\mathrm{Q}=\mathrm{KSi}$ (où $\mathrm{K}$ est la perméabilité mal connue ici et $\mathrm{S}$ la section offerte à l'écoulement, en admettant pour $\mathrm{K}$ une valeur moyenne représentative de l'ensemble de la surface $\mathrm{S})$.
L'évolution de cette ligne sur la figure 6, permet de considérer la différence entre les piézomètres 23 et 18 comme représentative de la pente de l'écoulement. Cette différence est choisie de préférence à celle donnée par les pièzomètres 19 et 23 . Ce sont surtout les deux piézomètres 18 et 23 , intégrant une assez grande distance, sans toutefois être trop perturbés par des variations topographiques du sillon rocheux, qui sont suivis le plus régulièrement et font l'objet d'une attention continue. Ce n'est qu'en amont du piézomètre 18 que le sillon devient étroit et que la pente varie d'une façon importante.

4.3 La figure 7 donne une courbe de corrélation entre la différence $\Delta h$ entre les niveaux des piézomètres 18 et 23, c'est-à-dire la pente de l'écoulement ou le gradient, et la dénivelée totale $\Delta \mathrm{H}$ c'est-à-dire la différence entre les niveaux amont moins aval.

Comme le sillon varie avec la section qu'il offre à l'écoulement, lorsque la nappe aval ou l'ensemble des piézomètres varient de cote, il a fallu ramener ces $\Delta$ h à des valeurs considérant la nappe aval comme constante. Les cotes aval varient entre 648 et 652 , on adopte une cote moyenne de 650 et on ramène toutes les lectures à cette cote. Lorsque la nappe aval monte, la section offerte à l'écoulement croît et à débit égal, le $\Delta$ h diminue. 


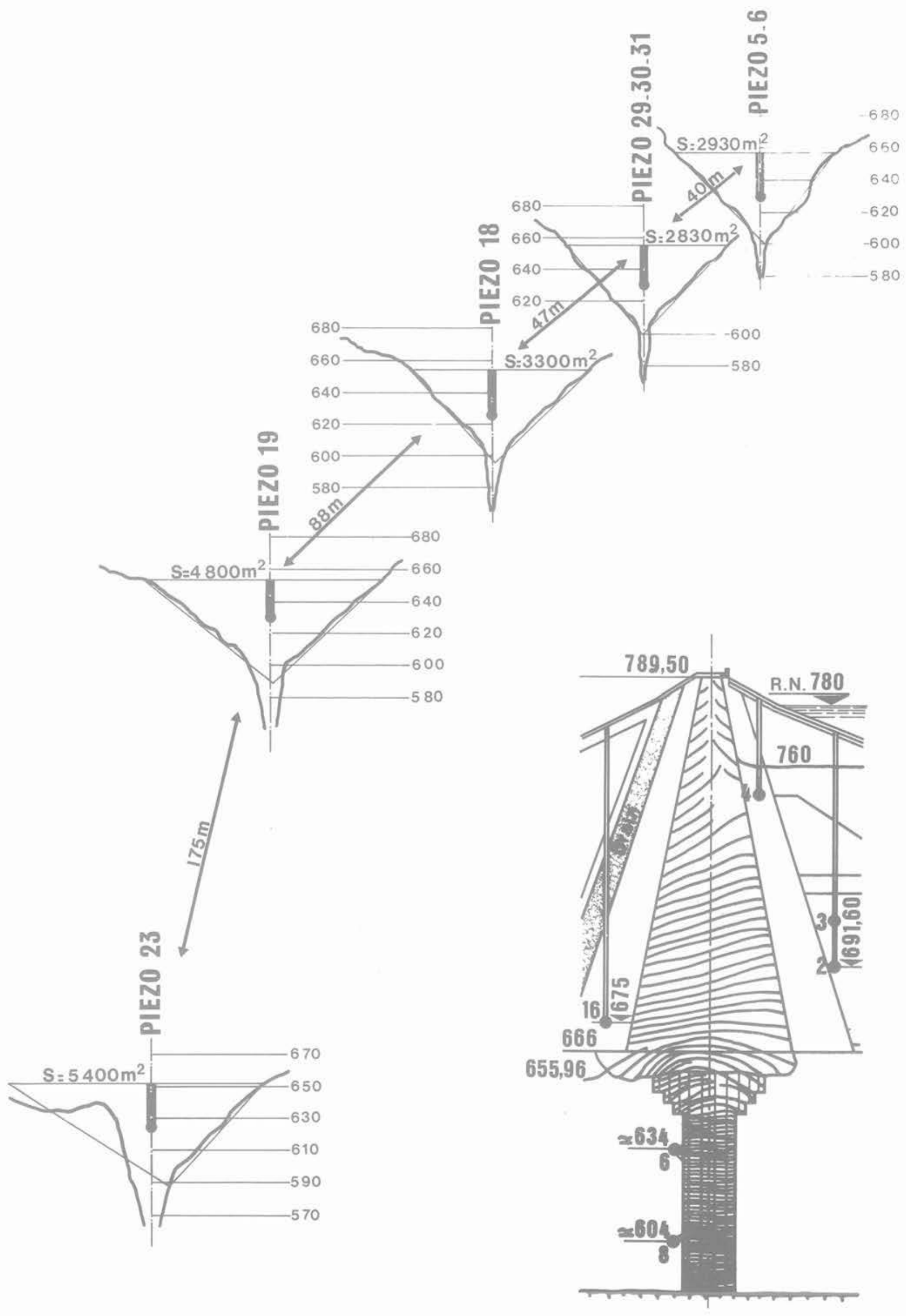

Fig. 3 


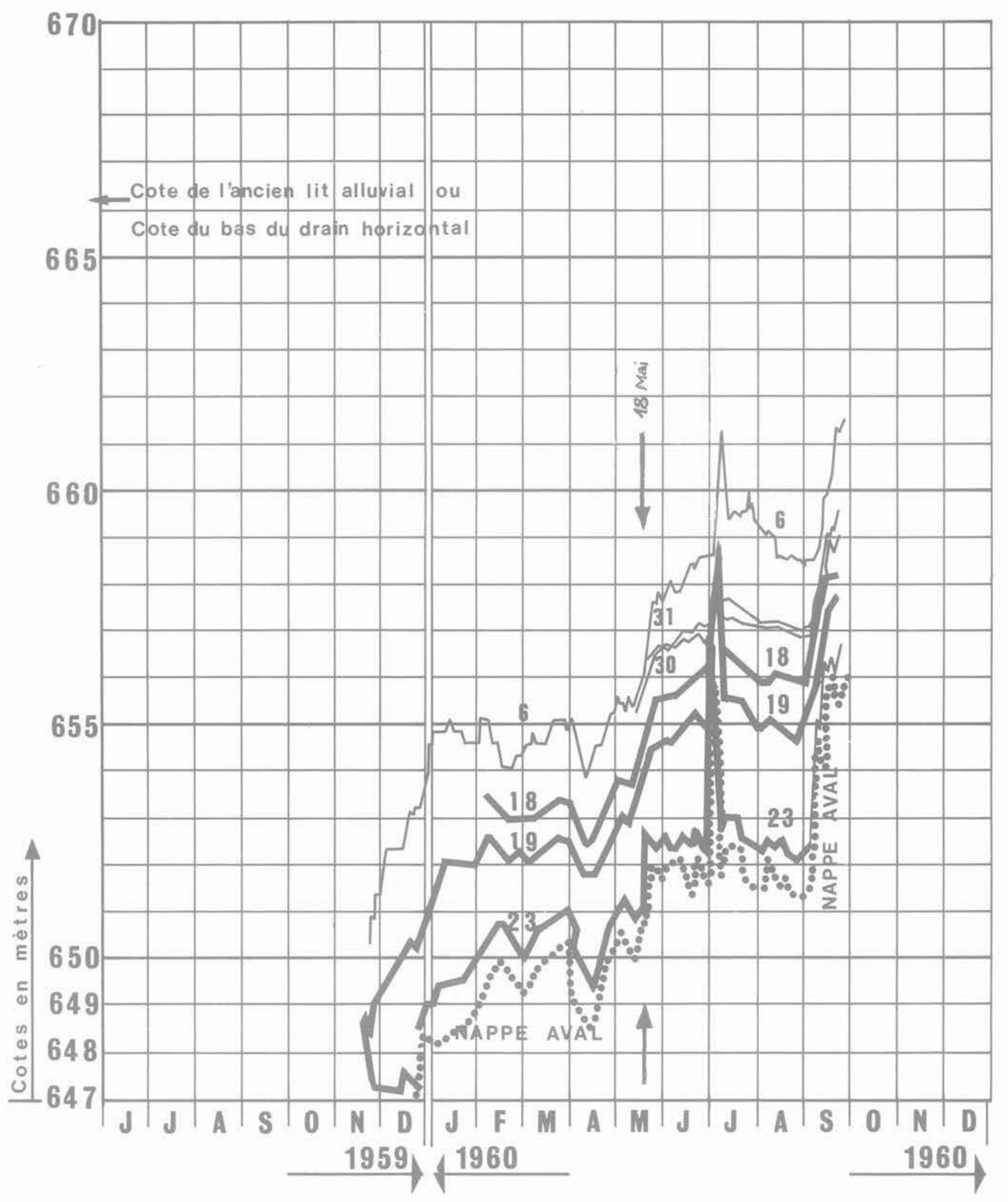

Fig. 5 Piézomètres aval (en fonction du temps) 


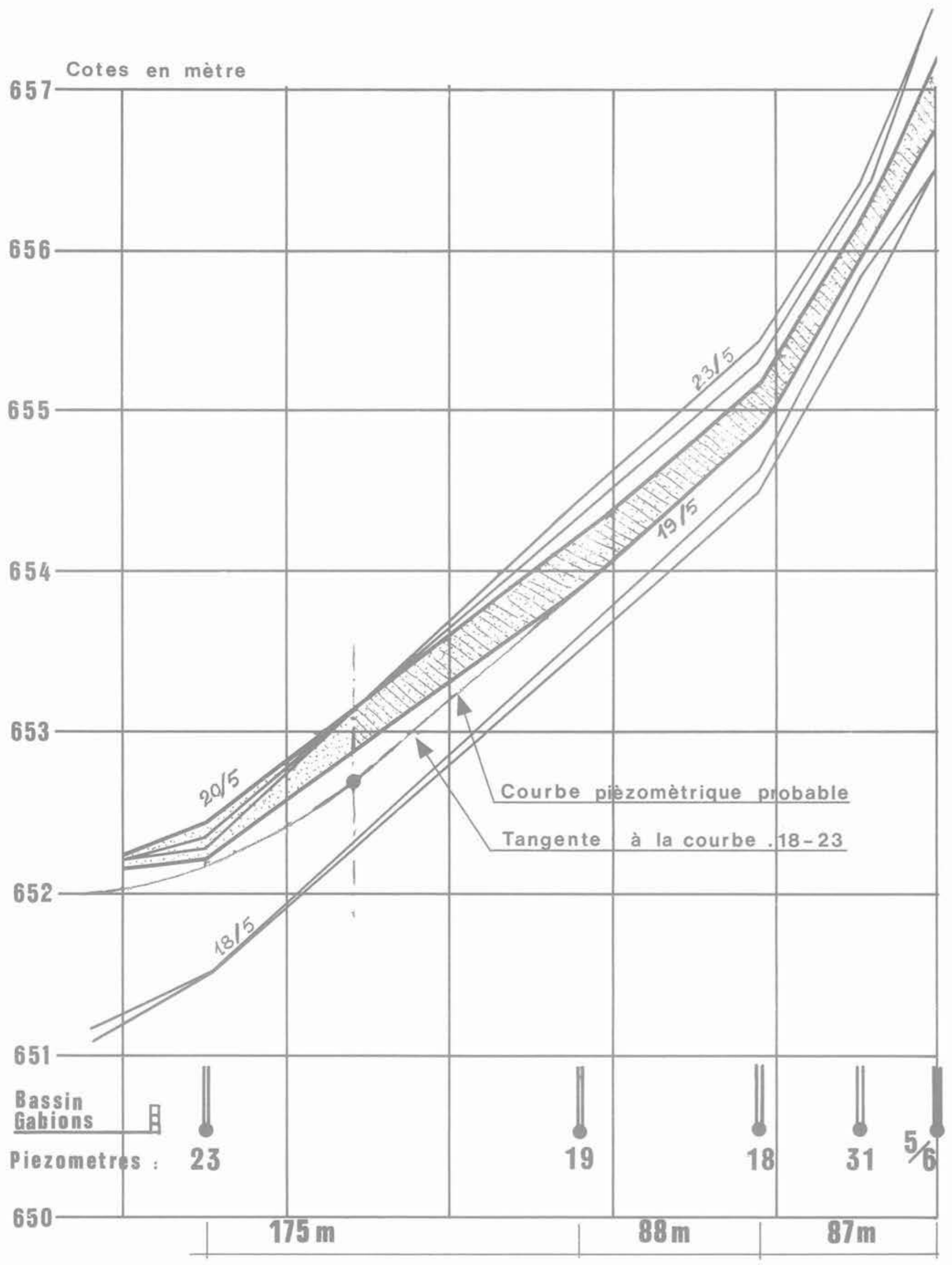

Fig. 6 Courbe piézométrique dans les alluvions aval (de la coupure au bassin de compensation) 
La section de passage au droit du piézomètre 23 à la cote 652 est d'après les relevés de sondages réalisés en reconnaissance par Solétanche (voir fig. 3) de $5400 \mathrm{~m}^{2}$ pour une largeur de nappe de 169 mètres.

Au piézomètre 18 on obtient pour une cote de 655 respectivement $3300 \mathrm{~m}^{2}$ et 112 mètres.

C'est-à-dire que si la nappe aval monte de $1 \mathrm{~m}$, l'augmentation relative de la surface est égale: $\Delta S / S=169 / 5400=3,1310^{-2}$ pour la section au droit du piézomètre -23 - et $\Delta S / S=112 / 3300=3,4010^{-2}$ pour la section au droit du piézomètre 18 .

Un chiffre moyen de $3,3 \%$ est retenu, c'est-à-dire que l'augmentation de niveau aux piézomètres 18 et 23 est inférieure à $1 \mathrm{~m}$ après stabilisation :

le gradient $\mathrm{i}=\Delta \mathrm{h} / \Delta \ell$ devient, comme $\mathrm{Q}=\mathrm{Ki}_{0} \cdot \mathrm{S}=$ $\mathrm{KiS}(1+\Delta \mathrm{S} / \mathrm{S})$

$\mathrm{i}=\mathrm{i}_{0} /(1+\Delta \mathrm{S} / \mathrm{S})$ soit pour $\mathrm{x}$ mètre $\left.: \mathrm{i}=\mathrm{i}_{0} /(1+\Delta \mathrm{S} / \mathrm{S}) \cdot \mathrm{x}\right)$.

La courbe de corrélation (fig. 7) est définie à cote aval constante, les lectures étant ramenées à 650 .

Cette courbe, à part les fluctuations inévitables, telles que celles résultant de la baisse du plan d'eau aval qui entraine un essorage du terrain, donc un débit apparent supérieur, ou de la montée de la même cote aval qui entraîne un relèvement général des piézomètres : donc une perte de débit pour remplir les vides du matériau, c'est-à-dire un débit apparent d'écoulement plus faible, est bonne. Mais cette corrélation n'est pas une droite, on reparlera de la forme de cette courbe plus loin.

Entre le 19 et le 23 mai 1960, la cote amont a varié de $80 \mathrm{~cm}$, c'est-à-dire à peine $1 \%$ de la charge, on néglige cette augmentation et on suppose que la nappe aval (ramenée à 650) n'a pas changé non plus.

Le 23-5, le $\Delta h$ était de $3,34 \mathrm{~m}$,

le $20-5$, le $\Delta$ h était de $2,86 \mathrm{~m}$,

le $19-5$, le $\Delta$ h était de $2,80 \mathrm{~m}$.

Comme le 23 mai, la stabilisation haute n'est pas encore acquise, il convient (en se référant aux périodes stables du mois d'août 1960, en figure 5) de retenir un $\Delta \mathrm{h}=3,50 \mathrm{~m}$ au lieu de $3,34 \mathrm{~m}$ (entre les piézomètres 18 et 23) comme pente capable d'écouler le débit total.

Le 19 et le 20 mai la nappe indique un débit moindre : l'écoulement suivant la loi de Darcy est à faible vitesse et la pente de la nappe représente le débit.

La perte de débit, alors que les fuites sont supposées constantes ne peut servir qu'à remplir les alluvions qui se trouvent noyées par l'augmentation du niveau de la nappe.

Le débit stocké dans les alluvions entre le 19 et le 20 mai est donc, si $Q$ est le débit total cherché :

$Q(1-2,80 / 3,50)=0,20 Q$ le 19 mai,

$Q(1-2,84 / 3,50)=0,19 Q$ le 20 mai.

Le volume dans lequel a été stockée cette quantité d'eau est compris entre les deux surfaces libres du 19 et du 20 mai dont la section est grisée sur la figure 6 . La limite amont est celle de la coupure étanche. La limite aval doit être interpollée comme étant celle où la courbe piézométrique réelle (que l'on doit imaginer entre les 3 points mesurés) possède une tangente représentative de la vitesse d'écoulement prise en compte le 19 ou 20 mai, parallèle à la droite piézométrique 18-23. En effet, à l'aval de ce point, la vitesse est plus faible, attestant d'un volume d'eau stockée encore plus grand. Cette limite aval est évidemment très imprécise, mais l'erreur ne peut dépasser 10 à $15 \%$.
Le volume de terrain qui va être noyé donc rempli par l'eau stockée est représenté par la surface hachurée de la figure 6 et ceci pour une période de 24 heures où les conditions sont restées relativement stables. Ce volume calculé donne $11580 \mathrm{~m}^{3}$ d'alluvions.

4.4 Les alluvions en place ont un certain volume de vides. On donne des chiffres de densité sèche de l'ordre de 2,050 à 2,100 (plutôt 2,050), avec un poids spécifique des grains de 2,74 et pour une teneur en eau de $2 \%$ teneur en eau admise pour les cailloux seuls). Nous avons alors, si $\mathrm{V}$ est le volume des vides disponibles et $\mathrm{V}^{\prime}$ le volume total :

$V=n V^{\prime}=V^{\prime} \quad((2,740-2,050) / 2,740-2,050 \times 2 / 100)=$ $0,21 \mathrm{~V}^{\prime}$ avec 2,100 on aurait $\mathrm{V}=0,19 \mathrm{~V}^{\prime}$.

On prend un chiffre moyen V égal à $20 \%$ de $V^{\prime}$ il est à noter que le dossier technique de 1955 donne une densité saturée des fondations de 2,36 ou 2,37 ce qui là encore donne :

soit $\mathrm{V}$ grains $=(2,36-1) /(2,74-1)$

$V$ grains $=1,36 / 1,74=0,78$.

II y a donc $22 \%$ d'eau en volume soit là encore $20 \%$ d'eau libre (le reste étant lié aux cailloux).

Entre le 19 et le 20 mai soit en 24 heures, le volume d'eau stockée dans la zone hachurée de la figure 6 a été :

$11580 \times 0,20=2316 \mathrm{~m}^{3}$

à raison de 0,195 du débit stocké (moyenne des deux chiffres cités plus haut) le débit $Q$ total traversant l'ensemble noyau coupure rocher sera :

$Q=\left(231610^{3} / 0,195\right) /(24 \times 3600)=137$ litres $/$ seconde.

A 60 m à l'amont, du piézomètre 23, la section offerte à l'écoulement est de $5200 \mathrm{~m}^{2}$. Le gradient de circulation est 3,50/260 (moyenne entre les cotes des piézomètres 18 et 23 ) en période d'écoulement stabilisé. La perméabilité moyenne des alluvions en place peut s'écrire :

$K=(0,137 / 5200) \times(260 / 3,50)=2 \cdot 10^{-3} \mathrm{~m} / \mathrm{s}$.

On trouve donc $137 \mathrm{l} / \mathrm{s}$ à $60 \mathrm{~m}$ à l'amont du 23 , entre les piézomètres 18 et 31 on trouverait :

$2 \cdot 10^{-3} \times(0,97 / 47) \times 3100=130 \mathrm{l} / \mathrm{s}$

aux approximations près, on voit que ce chiffre est comparable au précédent.

S'il doit y avoir une influence du rocher, il y a tout lieu de croire que tout se passe très près de la coupure en aval (rocher, contact, coupure...).

Évidemment, ce débit et la perméabilité trouvée dépendent beaucoup de la porosité utile (quantité d'eau libre capable de circuler dans le matériau). On a considéré que seuls $2 \%$ de l'eau restaient liés aux cailloux et que la totalité de l'air se trouvait chassée par l'eau pénétrant très lentement en vitesse réelle.

II est ici très difficile de parler du coefficient faisant intervenir le "fluide mort" car le matériau a eu le temps de s'essorer en 3 ans au moins et se trouve de nouveau rempli d'eau, on a retenu $10 \%$ du volume total des vides comme étant occupés par du " fluide-mort "

Évidemment, on peut prendre plus, entre 20 et $50 \%$ cite-t-on. Dans ces conditions le débit serait soit 100 litres soit même 70 litres/s. Le laboratoire de Mécanique des Sols de Grenoble lors d'essais de remplissage d'un sable (dans le cadre d'un écoulement non permanent, à propagation à partir d'un puits) a 


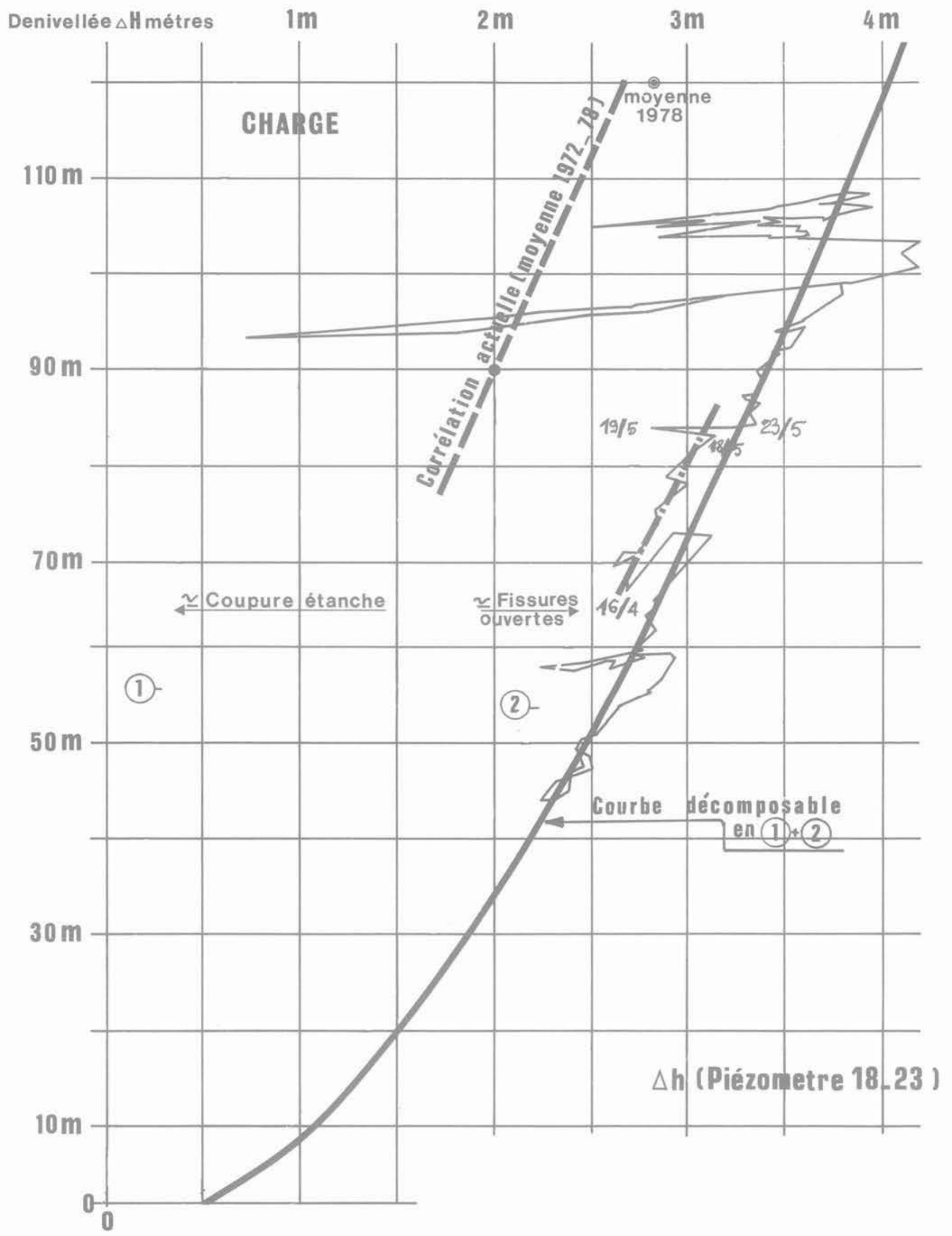

N.B. Lectures ramenées à

la cote aval constante 650

Fig. 7 Corrélation entre la différence des piézomètres $18-23$ et la charge totale 
donné un remplissage de 92 à $93 \%$ du volume des vides avec une porosité de l'ordre de $30 \%$. Pour les matériaux de Durance, plus grossiers et très propres, le remplissage se doit d'être plus complet. On peut penser qu'un chiffre de 120 litres est sage (la perméabilité moyenne n'en est pas affectée). La précision de ce résultat, bien qu'un calcul d'erreur précis soit impossible, se situe à moins de $30 \%$ alors qu'un calcul partant de la perméabilité est souvent faux d'une puissance de 10 ou plus.

Comme ce phénomène, de diminution apparente du débit lors d'une montée d'eau donc d'un remplissage des alluvions est à peu près identique à chaque modification brutale du plan d'eau aval, on doit pouvoir croire à la valeur des chiffres avancés.

Ces chiffres, demandent à être prouvés, mais cette méthode de calcul donne un idée sur la possibilité que I'on peut avoir d'évaluer les fuites d'un barrage en terre posé sur un sillon d'alluvions avec une assez bonne précision, après avoir vérifié en laboratoire l'influence du "fluide mort" et de I'air, c'est-à-dire la maniére dont se fait le remplissage des alluvions par l'eau.

4.5 Dans cet essai, non programmé, des faits favorables se sont rejoints, car la variation du niveau aval fut brutale et d'une amplitude correcte (pas trop grande, car les variations sont alors difficilement exploitables avec basculement de la nappe et pas assez de piézomètres, et pas trop petite car l'erreur de lecture devient prépondérante). II y a eu aussi, et c'était nécessaire, une stabilisation aval avant et surtout après la modification brutale du niveau (voir $\$ 4.1$ ). On peut, partant de là, moyennant une reconnaissance du sillon rocheux correcte (roches et alluvions) au droit de deux piézomètres aval distants de telle sorte que l'on puisse lire une différence de niveau de l'ordre de $2 \mathrm{~m}$ environ (pour tenir compte de la précision de la mesure et de la différence de débit à trouver), disposer, pour les barrages munis d'un ouvrage de compensation à niveau variable d'une façon relativement rapide, d'une «station de jaugeage" pour mesurer les fuites du barrage.

On peut remarquer que la nappe aval, s'écoulant sous la digue a une pente faible (de l'ordre de $2 \%$ ) : les équipotentielles restent à peu près verticales, c'est dire que l'écoulement, même à la partie supérieure de cette ligne modifiée par les conditions d'aval, est presque parallèle à la surface. II y a tout lieu de croire que toute modification de la pente (plus ou moins $30 \%$ ) traduit une modification du débit circulant au-dessous du point envisagé.

\section{Nature des fuites}

5.1 La courbe de corrélation représentée en figure 7 , ne passe pas par zéro pour une charge nulle.

La courbe finale trouvée serait l'addition (puisque dans les alluvions la loi de Darcy est valable) d'un écoulement fonction linéaire de la charge, donc régi par la loi de Darcy, c'est-à-dire un écoulement en milieu poreux avec de faibles vitesses et d'une écoulement plus rapide dans les passages. plus importants, donc plus turbulents et régis par une loi de débit fonction de la racine carrée de la charge. Ce serait plutôt de la charge à une puissance comprise entre $1 / 2$ et 1 . Cet écoulement peut même être variable en fonction du temps, d'abord à cause des injections et ensuite à cause de nouveaux cheminements dans le rocher intéressés par la montée de l'eau.

La décomposition de la courbe de débit en fonction de la charge décrite en figure 7 est donc très arbitraire et ne donne qu'une idée fictive de l'ensemble de l'écoulement. Ce que l'on peut voir et c'est là le point à retenir, c'est que tout le débit ne vient pas à travers des zones injectées de la coupure et du rocher, il y a de l'ordre de $90 \%$ de l'écoulement qui n'est pas régi directement par loi de Darcy c'est-à-dire avec écoulement proportionnel à la charge.

Si au lieu de la courbe en racine carrée de la charge on en prend une autre (moyenne d'écoulement compris entre le turbulent et le laminaire) on peut y ajouter une fonction linéaire de Darcy plus importante que 10\% pour obtenir l'équivalence de la courbe décrite en figure 7.

Mais si les $10 \%$ considérés sont dus à la seule coupure étanche dans sa partie profonde, donc de largeur constante, les gradients dans la coupure $(90 / 15=6)$ et juste après la coupure $(1 / 40)$ permettent de définir un gain de perméabilité réalisé par la coupure de :

$\mathrm{K}$ alluvions $/ \mathrm{K}$ coupure $=6 \times 40 / 1 / 10=2400$.

On peut rappeler que lors des plots d'essais réalisés dans la coupure un chiffre de 1000 donc du même ordre que celui-ci avait été trouvé.

La perméabilité de la coupure serait donc de l'ordre de :

$2 \cdot 10^{-3} / 2000=10^{-6} \mathrm{~m} / \mathrm{s}$.

5.2 Entre les dates du 16 avril et du 18 mai 1960, un autre phénomène est venu perturber la courbe de corrélation citée (fig. 7). Le niveau du piézomètre 34 situé à l'aval du piézomètre 23 est devenu plus haut que le piézomètre 23 de l'ordre de $15 \mathrm{~cm}$. Logiquement, en cas d'homogénéité, le 34 doit être plus bas de $15 \mathrm{~cm}$ par rapport au 23. En même temps que cela le débit apparent (différence $\Delta h$ entre les piézomètres 18 et 23) a baissé de l'ordre de $6,25 \%$. Nous pouvons croire que ces $6,25 \%$ sont passés en amont du piézomètre 18 pour alimenter le piézomètre 34 par le fond du sillon (réputé très perméable car très hétérogène). Depuis la prise du piézomètre 34 , l'écoulement se fait soit par remontée dans le bassin de compensation, soit par écoulement toujours en profondeur dans le sillon.

Considérant une section intéressée par cet écoulement de l'ordre de $600 \mathrm{~m}^{2}$ (au-dessous de la cote 600 , il y avait un socle rocheux à la cote 600 lors de la perforation du 34 ). Le débit aurait doublé à peu près durant cette période c'est-à-dire que la perméabilité serait deux fois plus forte dans cette partie alluviale soit $4 \cdot 10^{-3} \mathrm{~m} / \mathrm{s}$. Suivant les chiffres donnés par les études préliminaires (dossier technique E.D.F. 1955) la perméabilité varie de $10^{-3}$ à $10^{-5} \mathrm{~m} / \mathrm{s}$ avec des maxima au contact lias-remplissage allant jusqu'à $2 \cdot 10^{-2} \mathrm{~m} / \mathrm{s}$.

Ces chiffres élevés, au contact, doivent faire regarder de plus près les piézomètres 31 et 30 . Tous deux sont à la même distance de la coupure théorique et malgré cela le piézomètre 31 (côté R.D.) s'est trouvé $18 \mathrm{~cm}$ puis $40 \mathrm{~cm}$ plus haut que le piézomètre 30 . On peut alors considérer qu'un assez gros débit vient du contact avec le rocher du lias et le remplissage côté R. D. Le rocher R. D. par lui-même à l'air d'être sain et les injections jusqu'à la cote 600 n'ont pas modifié de beaucoup ce phénomène. II reste le contact qui peut être, comme nous l'avons vu de l'ordre de $2 \cdot 10^{-2} \mathrm{~m} / \mathrm{s}$, $n$ 'aurait pas retenu l'injection et offrirait une grande 
CORRELATION DU DEBIT DU DRAIN (Galerie R.G.)

Avec la cote de l'eau a l'amont
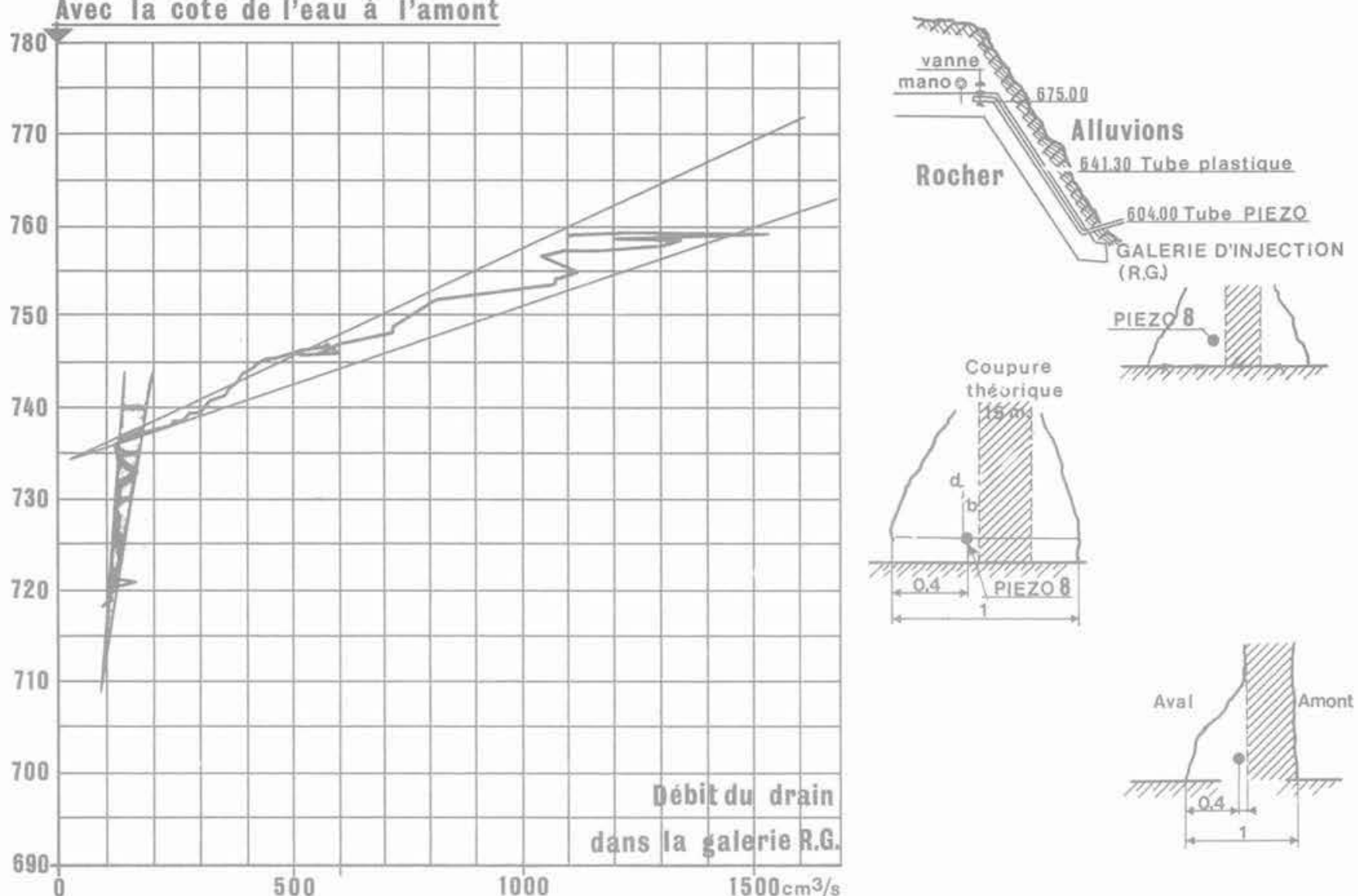

Fig. 8

Fig. 9

possibilité d'écoulement. Par son effet de drainage de la zone injectée on pourrait avoir un débit qui ne serait plus fonction de la charge seule mais de la charge à une puissance inférieure à 1.

Par rapport aux chiffres avancés dans le dossier technique et ceux retrouvés ici, un bon accord existe au point de vue perméabilité aussi bien pour la coupure étanche, qu'en ce qui concerne les alluvions en place. Au point de vue débit total, la nappe était estimée en 1955 à $200 \mathrm{l} / \mathrm{s}$ et sa pente d'écoulement au droit des mêmes piézomètres (18 et 23) était plus forte. Le débit global de percolation trouvé ici après mise en eau est lui aussi en bon accord avec les estimations. origines.

5.3 II y a bien sûr, outre les $\propto$ fissures ouvertes , des phénomènes de déversement à partir de certaines cotes. La figure 8 représente un drain mesuré en R. G., au Serre De Monge dont la cassure brutale de la courbe de corrélation montre un débit non proportionnel à la charge, mais pas forcément hors de l'application de la loi Darcy puisqu'il semble linéaire à partir de la cote 735 .

5.4. A l'aval de la coupure, le piézomètre $n^{\circ} 8$, (dont on a pu mesurer le débit sous charge, en 1960, ce qui a permis de calculer une perméabilité de poche de l'ordre de $10^{-7} \mathrm{~m} / \mathrm{s}$ ) doit se trouver dans une extension de la coupure étanche entraînée par le courant lors de l'exécution (voir fig. 9). Diverses analyses devaient conduire à supposer une certaine usure de cette excroissance supplémentaire de la coupure. Complété par trois autres forages, plus amont et aval, ce profil de la coupure se révèle stable.

\section{Remarques de synthèse}

6.1 On a donc, par une approche pratique et loin des études mathématiques des écoulements à régimes variables, pu calculer des débits relativement précis.

La perméabilité qui est une donnée de base d'habitude, mais qui souffre d'une grosse imprécision quant à sa valeur réelle en place, devient ici un chiffre que l'on peut déduire du débit. On retrouve de ce fait outre la puissance de 10, une certaine crédibilité de son chiffre significatif. On peut aussi comparer les chiffres trouvés avec ceux retenus dans l'étude préalable donnés en figure 10 .

6.2 En 1978, soit 18 ans après la première mise en eau, la courbe de corrélation citée en figure 7 note une moyenne correspondant à un débit de $40 \%$ plus faible. De plus, en exploitation normale, c'est-à-dire une charge $\Delta \mathrm{H}$ comprise entre 80 et $120 \mathrm{~m}$, la linéarité avec l'origine semble presque acquise. Le débit global a diminué corrélativement et se situe donc au-dessous de 100 litres par seconde à pleine charge.

Tout laisse prévoir un colmatage du fond à l'amont, ce qui est excellent. Le recharge amont du barrage, elle, doit rester très poreuse puisque les sédiments de surface apparents sont peu abondants. Ce sont donc les cheminements des écoulements plus rapides que ceux de Darcy qui se sont trouvés ainsi colmatés.

Tout se passe actuellement comme si les percolations provenaient de milieux, peut être de perméabilités différentes, mais homogènes et conformes aux milieux poreux où la loi de Darcy est applicable. 


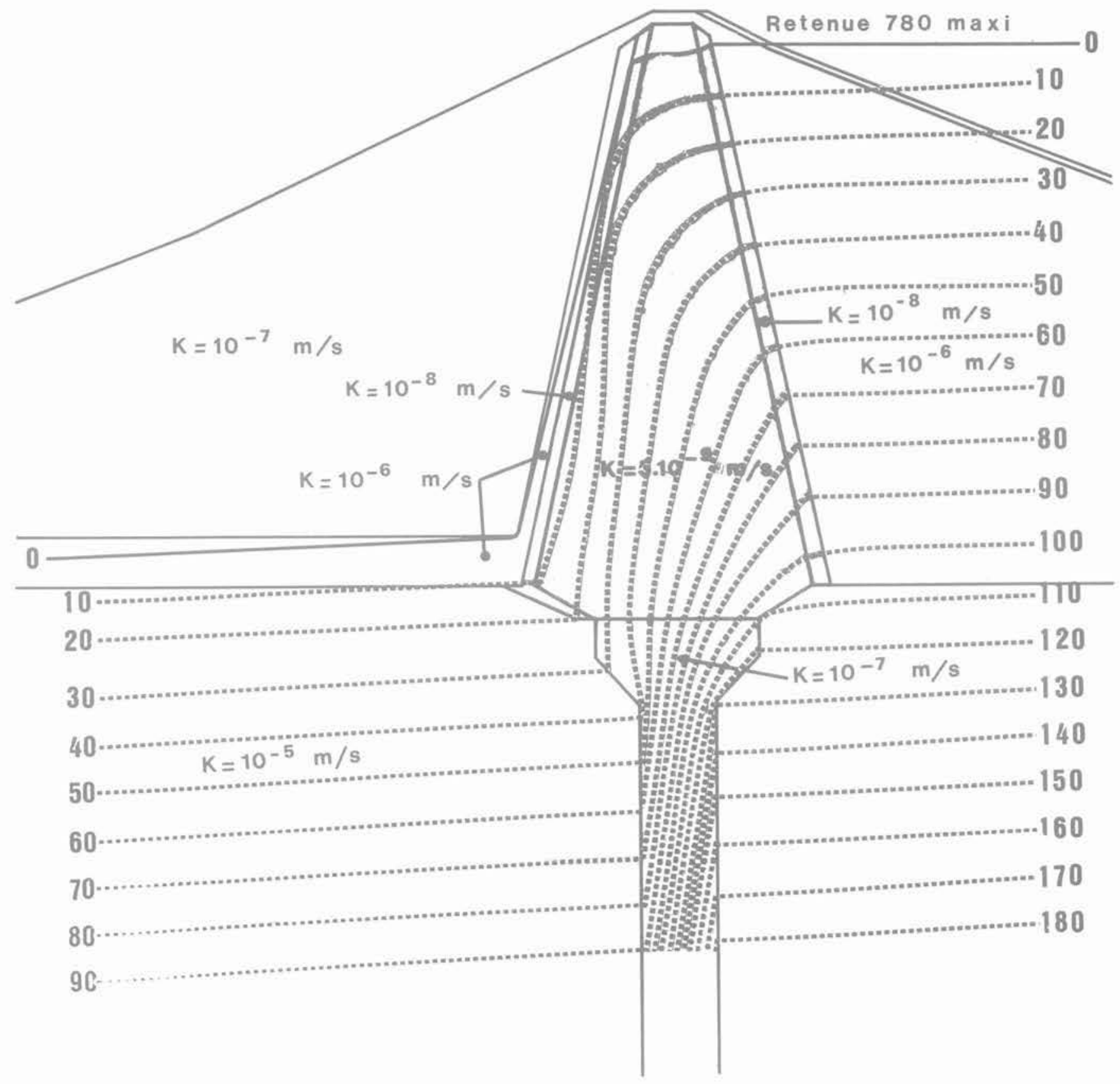

Fig. 10 Répartition des pressions (retenue pleine) par analogie électrique

\section{Références Bibliographiques}

E. Ledeuil - Contribution à l'étude des contraintes, des déformations et des circulations d'eaux dans le barrage de Serre-Ponçon. Thèse de Doctorat de spécialité 1960 Grenoble.

J. Barge - Ausculation de la digue de Serre-Ponçon. Annales de l'Institut Technique du Bâtiment et des T.P. $16^{\circ}$ année $n^{\circ} 189$ Septembre 1963.

J. Barge - G. Post - P. Huynh - Ausculation de la digue de Serre-Ponçon. $8^{\circ}$ Congrès International des Grands Barrages R3-Q29. Volume II - Edimbourg 1964.

P. Combelles - $12^{\circ}$ Congrès International des Grands Barrages Mexico 1976. Discussion Q45 - Volume V, p. 290. 